CULTURA, LENGUAJE Y REPRESENTACIÓN / CULTURE, LANGUAGE AND REPRESENTATION · ISSN 1697-7750 • VOL. XV \2016, PP. 81-98 REVISTA DE ESTUDIOS CULTURALES DE LA UNIVERSITAT JAUME I / CULTURAL STUDIES JOURNAL OF UNIVERSITAT JAUME I

DOI: HTTP://DX.DOI.ORG/10.6035/CLR.2016.15.6

\title{
La radiotelevisión pública local en Andalucía: situación actual e incidencia social. Análisis cuantitativo y cualitativo de las emisoras de la provincia de Cádiz ${ }^{*}$
}

\author{
Local public broadcasting in Andalusia: present situation \\ and social impact. Quantitative and qualitative analysis \\ of the radio stations in the province of Cádiz
}

RESUMEN: Este artículo tiene como principal objetivo la presentación de los resultados obtenidos en el Proyecto del Plan Nacional I+D+I CSO 2011-29195: La radiotelevisión pública local en Andalucía: situación actual e incidencia social. El proyecto tiene como objetivos principales: 1) conocer la realidad de los medios de comunicación públicos locales (radios y televisiones locales) de la comunidad andaluza, para comprobar en qué medida estos medios sirven para propiciar una mayor participación ciudadana; 2) conocer los contenidos que ofertan las emisoras municipales de radio y televisión en Andalucía, teniendo en cuenta, preferentemente, aquellos que responden a los criterios de servicio público. En este sentido, se presentarán los resultados obtenidos, correspondientes a la provincia de Cádiz. En el nivel cuantitativo se ha elaborado un cuestionario dividido en seis grandes apartados (ficha técnica, programación y contenidos, recursos humanos, equipamiento e instalaciones y financiación) que se ha administrado telefónicamente a los responsables de cada una de las emisoras de la provincia. En el plano cualitativo, se ha aplicado la metodología del estudio de casos para analizar cuatro emisoras representativas de las radios públicas locales gaditanas. A la vista de los resultados, una de las principales conclusiones del estudio apunta a

* $\quad$ Este artículo se encuadra dentro del Proyecto del Plan Nacional I+D+I CSO 2011-29195 del Ministerio de Economía y Competitividad: La radio y la televisión local en Andalucía: situación actual e incidencia social, dirigido por Dr. Manuel Chaparro Escudero (IP). 
la posibilidad de considerar a estos medios públicos locales como termómetros de la calidad democrática de la sociedad andaluza, en general, y de las localidades en las que se ubica cada uno de estos medios, en particular. Los medios públicos locales, también denominados medios del tercer sector, se sitúan en un juego de fuerzas que amplia o limita las posibilidades de participación y de deliberación de la ciudadanía. Los resultados del proyecto apuntan en varios sentidos: 1) a un predominio de las fuerzas del mercado a la hora de orientar las políticas y las prácticas comunicativas de los medios públicos locales de Andalucía; 2) a una infrautilización de los medios públicos locales por parte del poder político local, que no termina de aprovechar las potencialidades de servicio público y de participación ciudadana de estos medios; 3) a un escaso empoderamiento comunicativo de la ciudadanía (Chaparro, 2007), que solamente en un reducido número de las localidades estudiadas está aprovechando estos medios para ejercer cotidianamente sus derechos en el campo comunicacional.

Palabras clave: medios públicos locales, tercer sector de la comunicación, análisis cuantitativo y cualitativo.

ABSTRACT: The main objective of this article is to present the results obtained in the Project of the National Plan I + D + I CsO2011-29195: Local public broadcasting in Andalusia: present situation and social impact. The project, which bears the same title as the present paper, has as main objectives: 1) know the reality of the local public media (local radio and television) of Andalusia, to check to what extent they serve to promote a greater citizen participation; 2) know the contents that the local radio and television offer in Andalusia, considering preferably, who answer the criteria of «public service». In this sense, we will present the results obtained in the province of Cadiz. At the quantitative level there a questionnaire has been developed and divided in six main sections (Specification sheet, programming and contents, human resources, equipment and facilities and financing) that has been administered by telephone to the responsible for each of the radio stations in the province. In qualitative terms, a case study methodology has been applied to analyze four representative stations of local public radio of Cadiz. In view of the results, one of the conclusions is the possibility of considering these local public media as thermometers of democratic quality of Andalusian society in general, and the locality where each is located such media, in particular. The local public media, also called Third Sector media, are placed in a game of forces which broad or limit the possibilities of citizens' participation and deliberation. The results of the project point to several directions: 1) a predominance of market forces at the time of guide policies and communicative practices of local public media of Andalusia; 2) a underuse of local public media by the local political power, which not ending exploit the potentials of public service and citizen participation of this medias and 3) a poor «communicative empowerment of citizenship» (Chaparro, 2007), which only in a small number of studied localities is leveraging these medias to exercise daily their rights in communication field.

Keywords: local public media, third sector of communication, quantitative and qualitative analysis. 


\section{Introducción}

En una definición canónica del concepto de tecnología, los autores de la teoría crítica planteaban que esta es un proyecto histórico-social en el que se proyecta lo que una sociedad, y los intereses en ella dominantes, tienen el propósito de hacer con las personas y con las cosas. ${ }^{1}$ Para ellos, analizar una determinada tecnología (televisión, fotografía, radio) consistiría en identificar los deseos, las necesidades, pero sobre todo, los intereses que los poderes dominantes en un determinado sistema social están dispuestos a ofrecer a los ciudadanos que habitan en él.

Siguiendo con la metáfora de la proyección, podríamos preguntarnos, en el contexto del tema que nos ocupa, si los medios públicos locales de Andalucía (preferentemente radios) son espacios en los que se proyectan tanto las posibilidades como las limitaciones que un determinado régimen democrático está dispuesto a ofrecer a la ciudadanía para su participación en la gestión de lo común.

De entrada, llama la atención, como lúcidamente apunta Cammaerts (2009), el resultado contradictorio que surge del análisis comparado de las políticas de comunicación que rodean a los medios comunitarios en el mundo en función del eje países occidentales-países en vías de desarrollo. Mientras que en los primeros (west) los medios comunitarios se ven desplazados a posiciones marginales, mantienen una larga tradición en la lucha por su derecho a existir, por conseguir frecuencias o por el reconocimiento político; asistimos a un contexto diferente en muchos países de África, Asia o Latinoamérica, en los que se implementan diversidad de iniciativas para intensificar la presencia de emisoras locales.

En este sentido, resalta el contraste que se produce al comparar el nuevo marco legislativo uruguayo (2007) y argentino $(2009)^{2}$ respecto a los medios comunitarios y sin ánimo de lucro, a los que se reserva un tercio del espectro, con la continua marginación y exclusión a la que estos mismos medios se han visto sometidos a lo largo de los más de treinta años de democracia en España. Mientras que el «fenómeno» argentino/uruguayo está creando una auténtica dinámica de contagio en otros países de la región, la realidad en el continente europeo, a pesar de los matices que puedan existir entre los diferentes países, entra dentro del diagnóstico previamente apuntado.

1. Habermas, J. (1986): Ciencia y técnica como ideología, Madrid, Tecnos. Traducción del original alemán Wissenschaft und Technik als «Ideologie» (1968), escrito con motivo del $70^{\circ}$ de Herbert Marcuse.

2. Véase, al respecto, los comentarios a la citada ley en el texto colectivo coordinado por Baranchuk, M. y Rodríguez Usé, J. (2011): «Ley 26.522. Hacia un nuevo paradigma en comunicación audiovisual», Universidad Nacional de Lomas y Autoridad Federal de Servicios de Comunicación Audiovisual. 
Sin pretender idealizar la realidad latinoamericana, sometida también a tensiones y ambigüedades, queremos centrar nuestra reflexión en torno a las diferentes fuerzas y dinámicas que se proyectan sobre los medios públicos locales en Andalucía, para hacer caer sobre ellos lo mejor (y también lo peor) de la democracia actual.

En cuanto a los aspectos metodológicos, la primera fase de la investigación se ha abordado desde una perspectiva cuantitativa. La herramienta metodológica utilizada ha sido la elaboración de un cuestionario con cinco secciones principales (ficha técnica, programación y contenidos, recursos humanos, equipamiento e instalaciones y financiación) que se administró por vía telefónica a todas las emisoras de radio y televisión locales de Andalucía. En la segunda fase de la investigación, abordada de forma cualitativa, se ha desarrollado la metodología del estudio de casos para analizar cuatro radios representativas de las emisoras municipales de la provincia.

\section{Enfoque del análisis}

En este apartado vamos a sintetizar algunas cuestiones de carácter teórico que nos permitan enmarcar la realidad actual de los medios públicos locales andaluces, en el juego de tensiones y fuerzas que se proyectan sobre ellos, para ampliar o reducir las posibilidades de estas emisoras de ser espacios de participación ciudadana y de servicio público.

En un esfuerzo de síntesis resaltamos las tres dimensiones que se muestran en la figura $n^{0} 1$ :

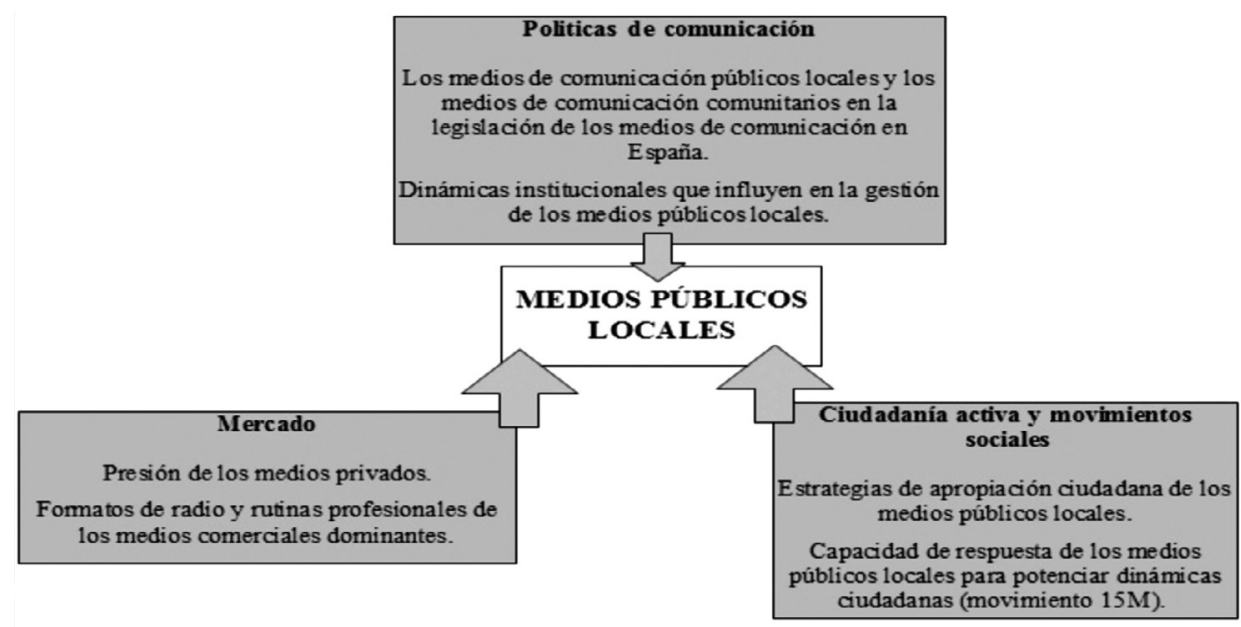

Fuente: elaboración propia

Figura 1. Dimensiones que afectan a los medios públicos locales 
1. Por un lado, las políticas de comunicación que, en el contexto español, se han venido elaborando desde el inicio del periodo democrático, especialmente en los últimos años del gobierno socialista de Zapatero, en las que se mantienen las constantes apuntadas al principio de este artículo: marginación y exclusión de los medios ciudadanos de los marcos de regulación, limitaciones técnicas y presupuestarias impuestas a estos medios o falta de reconocimiento, entre otros elementos. Junto a este nivel del análisis, centrado en cuestiones de carácter macroestructural, queremos focalizar nuestro trabajo, también, en otras dimensiones situadas, por decirlo coloquialmente, más a «ras del suelo», como pueden ser las inercias y dinámicas institucionales que, en cada localidad y en cada emisora, inciden en las posibilidades de aumentar el grado de participación ciudadana y de autogestión en cada medio. Estos factores pasan por el grado de independencia de los medios respecto de las directrices más o menos explícitas que llegan del poder político local, la autonomía y grado de decisión que tiene el personal que trabaja en la radio para establecer las líneas de acción, la capacidad del personal de la emisora de ejercer como comunicadores y dinamizadores culturales, etc.

2. En segundo lugar, como otro grupo de fuerzas que se proyectan e inciden sobre los medios públicos locales, se encuentran las fuerzas del mercado. Entre ellas destaca la presión e influencia que, como lobby fuertemente organizado, juega en el panorama español la Unión de Televisiones Comerciales Asociadas (UTECA). Se trata, como aparece en su sitio web, ${ }^{3}$ de una asociación constituida en 1998 con la finalidad primordial de defender y representar los intereses comunes de las televisiones comerciales en el ámbito nacional, comunitario e internacional. Su poder de influencia llega hasta tal punto que algunos analistas del tercer sector de la comunicación en España (Meda, 2012) han visto reproducidos los intereses de esta organización en el nuevo marco legislativo español que han pasado a denominar a esta nueva ley la ley UTECA. Del mismo modo que, en el apartado anterior, establecíamos un doble nivel (macro y micro), en este caso utilizamos de nuevo esta doble articulación para abordar en el análisis la presencia que ejerce sobre los profesionales de los medios públicos locales y sobre las propias audiencias el modelo comercial: el formato de radio al estilo de las principales emisoras nacionales e internacionales o el

3. Sitio web de UTECA: http://www.uteca.com/uteca/GestorWeb?id=21. 
modelo mainstream de producir espacios informativos, que lleva a reproducir en los medios públicos locales los mismos formatos y estilos de los grandes medios, pero con menos recursos y menor calidad, renunciando con ello a otros formatos y estilos comunicativos más coherentes respecto a la función social de estos medios.

3. Una tercera fuerza que incide sobre los medios públicos locales procede de la ciudadanía activa y de los movimientos sociales. En este nivel nuestro análisis pasa por estudiar esta relación desde cada uno de sus vectores o direcciones. Por un lado se encontrarían las estrategias de apropiación ciudadana con relación a estos medios públicos locales. En qué medida los ciudadanos y ciudadanas de una determinada localidad, a título individual u organizadamente (en asociaciones, ONG, movimientos sociales, sindicatos, etc.) ejercen su derecho de acceso a los medios y toman la palabra. En el otro sentido de esta relación, habría que analizar el grado de apertura y de receptividad que demuestran los gestores de estos medios públicos locales (y, en última instancia, el gobierno local) para abrir espacios a la participación ciudadana en el medio de comunicación y en la vida social, política y cultural de la localidad.

\section{El proyecto de investigación}

El proyecto de investigación titulado La radiotelevisión pública local en Andalucía: situación actual e incidencia social, tiene como principales objetivos los siguientes: ${ }^{4}$

1. Conocer e incidir en la realidad de los medios de comunicación públicos locales de la comunidad andaluza para contribuir a la vertebración territorial de Andalucía, así como propiciar una mayor y mejor participación ciudadana en los medios públicos de proximidad.

2. Disponer de un estudio comparativo que subraye las semejanzas y diferencias esenciales entre las emisoras públicas locales andaluzas de radio y televisión.

3. Conocer los contenidos, la independencia y la profesionalidad que ofertan las emisoras municipales de radio y televisión en Andalucía, así como su atención a los criterios de servicio público a la ciudadanía.

4. Tomado de la memoria técnica del proyecto. 
4. Evaluar las características y perspectivas de evolución y desarrollar propuestas dirigidas a mejorar su funcionamiento.

Durante el periodo 2011-2013 se ha llevado a cabo la primera fase del proyecto, cuantitativa, centrada en pasar un cuestionario a las emisoras de radio y televisión local en cada una de las provincias de Andalucía. La plantilla de recogida de datos que se ha utilizado está dividida en cinco secciones principales:

1. Hoja de datos en la que se solicitan los datos como el municipio y la comarca en la que se emite, la dirección postal, la dirección web, presencia en redes sociales, la frecuencia y la potencia del transmisor.

2. Programación y contenido, en la que cada estación desglosa las horas de programación según los porcentajes dedicados a la información, participación, cultura, deportes, etc.

3. Recursos humanos; sección centrada en la obtención de datos sobre el personal contratado, en los voluntarios de cada estación y su grado de formación académica o técnica.

4. Equipo e instalaciones, donde se pregunta a las emisoras por la potencia de emisión, los equipos disponibles (de radio, telefonía, informática), el tamaño de las instalaciones, etc.

5. Financiación, sección dedicada a conocer las diferentes fuentes de ingresos de cada emisora: aportación municipal, publicidad, patrocinio o donaciones, entre otros.

En la segunda fase del proyecto, la fase cualitativa, se desarrolló el estudio de casos. Esta fase tuvo lugar durante 2013. Para seleccionar los casos nos basamos en 4 criterios: la comarca a la que pertenecen, el tipo de gestión de la emisora, el partido político que gobierna en el municipio y el número de habitantes. Consideramos que a través de estos cuatro criterios se alcanza la suficiente representatividad de la diversidad de casos existentes.

Como observamos en la tabla 1 , respecto al criterio comarcal, cada emisora analizada pertenece a una comarca distinta. ${ }^{5}$ En cuanto al tipo de gestión, los casos seleccionados cubren los 3 tipos establecidos legalmente: gestión directa

5. La comarca es una demarcación territorial intermedia entre las provincias y los municipios, su importancia radica en que presentan unas características naturales, económicas y sociales comunes. Cádiz cuenta con 6 comarcas (Bahía de Cádiz, Campiña de Jerez, Campo de Gibraltar, Costa Noroeste de Cádiz, la Janda y Sierra de Cádiz). 
del Ayuntamiento, a través de la figura del patronato y mediante una empresa pública. Con el criterio del gobierno local se refleja la realidad que se da en la provincia, donde hay más emisoras públicas en funcionamiento en municipios gobernados por el PP. Atendiendo a la población se han analizado emisoras radicadas en núcleos urbanos con un alto índice de población (más de 200.000 habitantes como el caso de Jerez), así como emisoras situadas en poblaciones rurales de menos de 20.000 habitantes (como el caso de Ubrique y Trebujena). Con este criterio podemos observar la capacidad de incidencia social de las emisoras. Esto es, emisoras radicadas en localidades con mucha población se convierten en potenciales herramientas estratégicas de desarrollo social y de servicio público.

Tabla 1. Casos del estudio (Cádiz)

\begin{tabular}{lcccc} 
& Comarca & Tipo gestión & $\begin{array}{c}\text { Gobierno } \\
\text { local }\end{array}$ & $\begin{array}{c}\text { Población } \\
\mathbf{2 0 1 3} \text { (hab.) }\end{array}$ \\
$\begin{array}{l}\text { Radio San } \\
\text { Roque }\end{array}$ & $\begin{array}{c}\text { Campo } \\
\text { de Gibraltar } \\
\text { Radio Ubrique }\end{array}$ & $\begin{array}{c}\text { Empresa } \\
\text { pública }\end{array}$ & PSOE & 30.516 \\
$\begin{array}{c}\text { Se Cádiz } \\
\text { Onda Jerez }\end{array}$ & $\begin{array}{c}\text { Patronato } \\
\text { Campiña } \\
\text { Radio }\end{array}$ & $\begin{array}{c}\text { Empresa } \\
\text { pública }\end{array}$ & PP & 16.859 \\
$\begin{array}{l}\text { Radio } \\
\text { Trebujena }\end{array}$ & $\begin{array}{c}\text { Costa } \\
\text { de Cádiz }\end{array}$ & Ayuntamiento & IU & 211.900 \\
\hline
\end{tabular}

Fuente: elaboración propia

El trabajo de campo, dividido en dos momentos, consistió primeramente en una serie de entrevistas en profundidad, no estructuradas pero dirigidas por áreas temáticas. Siguiendo con la lógica de las tres fuerzas que actúan sobre las radios públicas locales (las políticas de comunicación, el mercado y la ciudadanía activa) se entrevistaron a los tres actores implicados: el responsable político de la emisora municipal, el directivo/a encargado de la gestión de la emisora y la ciudadanía-audiencia. Este enfoque pretende triangular y contrastar los intereses contrapuestos para poner de relieve el equilibrio de fuerzas 
que se proyecta en las emisoras. El segundo momento del trabajo cualitativo de campo abordó el análisis de contenido de aquellos programas radiofónicos que se enmarcan dentro del concepto de servicio público.

Para analizar los resultados nos guiamos por 5 indicadores propuestos por Gumucio (2006) que establecen un marco para definir los medios del tercer sector, aquellos que buscan la rentabilidad cultural y el desarrollo del entorno en el que se encuentran.

1. Que la propiedad de la emisora y de la frecuencia sea colectiva

2. Que exista participación de la comunidad en la toma de decisiones

3. Que exista un plan de sostenibilidad de la emisora

4. Que el $70 \%$ de los contenidos se generen localmente

5. Que exista pertinencia lingüística y cultural

\section{El trabajo de campo en la provincia de Cádiz}

Como se puede observar en la tabla 2, el censo actual de la provincia de Cádiz se compone de un total de 31 emisoras de radio, una cifra significativa teniendo en cuenta el tamaño (7.436 $\mathrm{km}^{2}, 1,47 \%$ de la superficie española) y la población de la provincia de Cádiz (1.230.594 habitantes). Basándose en el hecho de que, a nivel provincial, hay 44 municipios, se puede considerar como relevante el número de emisoras existentes en la provincia de Cádiz. Podemos decir que estos medios de comunicación públicos locales sirven para estructurar un territorio disperso y complejo como es la comunidad autónoma de Andalucía, en la que el $50 \%$ de la población mayor de 14 años vive en municipios con menos de 50.000 personas.

La tabla siguiente permite comprobar que las emisoras pertenecen en su mayoría a un entorno rural en el que por lo general operan casi exclusivamente medios de comunicación públicos. En este sentido, en la tabla 2 se puede ver que, de las 21 estaciones que actualmente permanecen abiertas en la provincia de Cádiz, 16 pertenecen a los municipios con menos de 50.000 habitantes, en los que no hay emisoras comerciales privadas, ya que estos municipios no son un mercado rentable. Sobre este aspecto, en la figura 2 se detalla la división demográfica por municipios: 
Tabla 2. Emisoras de radio pública local en la provincia de Cádiz

\begin{tabular}{|c|c|c|c|c|}
\hline Municipio & Emisora & $\begin{array}{l}\text { Año } \\
\text { inicio }\end{array}$ & Ema & $\begin{array}{l}\text { Abierta/ } \\
\text { cerrada }\end{array}$ \\
\hline 1. Alcalá del Valle & Radio Alcalá & 1994 & no & $\mathrm{a}$ \\
\hline 2. Arcos de la Frontera & Radio Arcos & 1982 & no & $\mathrm{a}$ \\
\hline 3. Barbate & Radio Barbate & 1986 & sí & $\mathrm{a}$ \\
\hline 4. Benalup & Benalup FM & 2011 & no & $\mathrm{a}$ \\
\hline 5 Benaocaz & Radio Benaocaz & - & no & c \\
\hline 6. Bornos & Radio Guadalete & 1995 & no & $\mathrm{a}$ \\
\hline 7. Cádiz & Onda Cádiz & 2007 & no & $\mathrm{a}$ \\
\hline 8. Castellar de la Frontera & Radio Castellar de la Frontera & - & no & c \\
\hline 9. Conil de la Frontera & Radio Juventud & 1987 & no & $\mathrm{a}$ \\
\hline 10. Chiclana & Radio Chiclana & 1985 & no & $\mathrm{a}$ \\
\hline 11. Chipiona & Radio Chipiona & 1982 & sí & $\mathrm{a}$ \\
\hline 12. El Bosque & Radio El Bosque & 1983 & no & $\mathrm{a}$ \\
\hline $\begin{array}{l}\text { 13. El Puerto de Santa } \\
\text { María }\end{array}$ & Radio El Puerto & 1999 & no & a \\
\hline 14. Grazalema & Radio Grazalema & 1990 & no & $\mathrm{a}$ \\
\hline 15. Jerez de la Frontera & Onda Jerez & 1986 & sí & $\mathrm{a}$ \\
\hline 16. Prado del Rey & Radio Prado del Rey & 1996 & no & $\mathrm{a}$ \\
\hline 17. Jimena de la Frontera & Radio Jimena & - & no & c \\
\hline $\begin{array}{l}\text { 18. La Línea de la } \\
\text { Concepción }\end{array}$ & Rtv. La línea & - & no & $\mathrm{a}$ \\
\hline 19. Los Barrios & Radio Los Barrios & - & no & c \\
\hline 20. Medina Sidonia & Radio Medina & - & no & c \\
\hline 21.Olvera & Radio Olvera & - & sí & c \\
\hline 22. Puerto Real & Radio Sol Puerto Real & - & no & c \\
\hline 23. Puerto Serrano & Radio Puerto Serrano & - & no & c \\
\hline 24. San Fernando & Radio Isla & - & sí & $\mathrm{a}$ \\
\hline 25. San Roque & Radio San Roque & - & sí & $\mathrm{a}$ \\
\hline 26. Sanlúcar de Barrameda & Radio Sanlúcar & 1986 & no & c \\
\hline 27. Tarifa & Radio Tarifa & 1995 & sí & $\mathrm{a}$ \\
\hline 28. Trebujena & Radio Trebujena & 1998 & sí & $\mathrm{a}$ \\
\hline 29. Ubrique & Radio Ubrique & 1984 & no & a \\
\hline 30. Vejer de la Frontera & Radio Vejer & - & no & $\mathrm{a}$ \\
\hline 31. Villamartín & Radio Villamartín & - & sí & c \\
\hline
\end{tabular}

Fuente: elaboración propia 


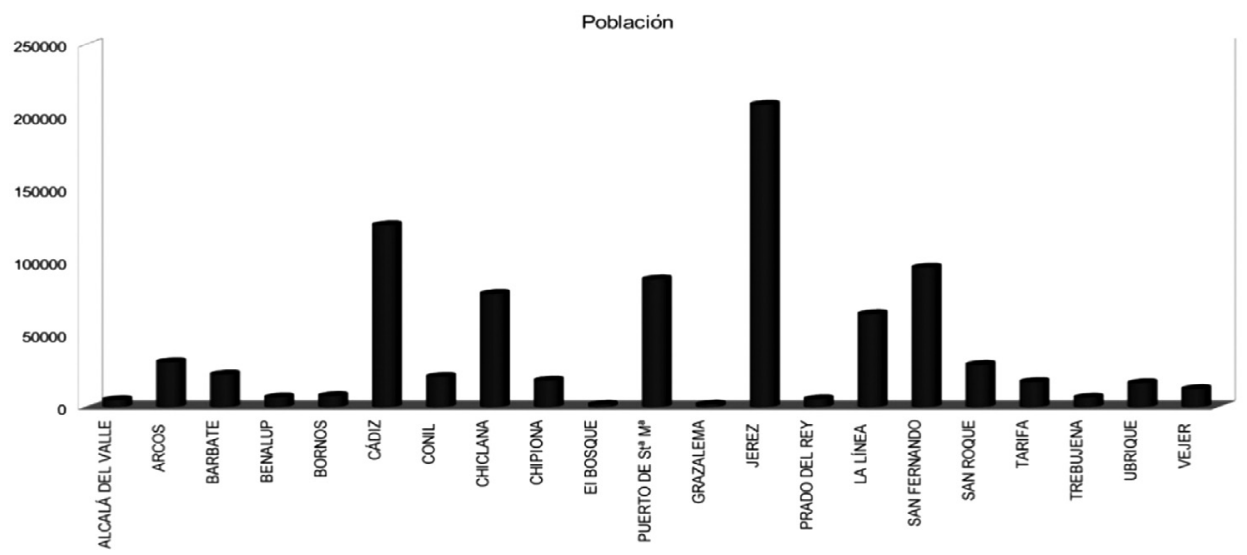

Fuente: elaboración propia

Figura 2. Población total por municipio, 2012

Una lectura de la tabla 2 permite comprobar que en la actualidad, en la provincia de Cádiz, hay 10 emisoras que están cerradas. Las razones que esgrimió el personal entrevistado de estas emisoras al ser preguntado por este hecho fueron las siguientes:

1. La crisis económica afecta a los municipios y estos, en sus políticas de ajuste, cierran la emisora del municipio.

2. El cambio de partido político en el gobierno de la localidad, que se produjo después de las últimas elecciones municipales (2011), ha provocado el cierre de la radio.

Si comparamos estos argumentos con el esquema inicial (el bloque de Política de comunicación) comprobaremos que las medidas adoptadas están estrechamente relacionadas con la carencia de una política de comunicación claramente definida en relación con este tipo de medios, de modo que se garantice su permanencia y estabilidad en el tiempo, al ser estos medios de proximidad una clara garantía de normalidad democrática. Por otro lado, en referencia al nivel micro de este bloque, se han identificado una serie de inercias y dinámicas institucionales que influyen en la gestión de estas emisoras: 
1. Dar prioridad a los recortes en política social y de servicio público (como es el caso de los medios públicos locales) antes que realizarlos en otras partidas presupuestarias.

2. Anteponer los intereses partidistas por delante de los proyectos municipales de largo alcance.

Esta cuestión queda reflejada en la figura 3, dedicada a los partidos políticos en el poder de aquellas localidades en que operan las radios públicas locales. En el caso de la provincia de Cádiz, cerca del $60 \%$ de estos municipios están gobernados por el Partido Popular, el $21 \%$ por el PSOE y el $16 \%$ por IU.

\section{Gobierno 2011}

20 radios abiertas
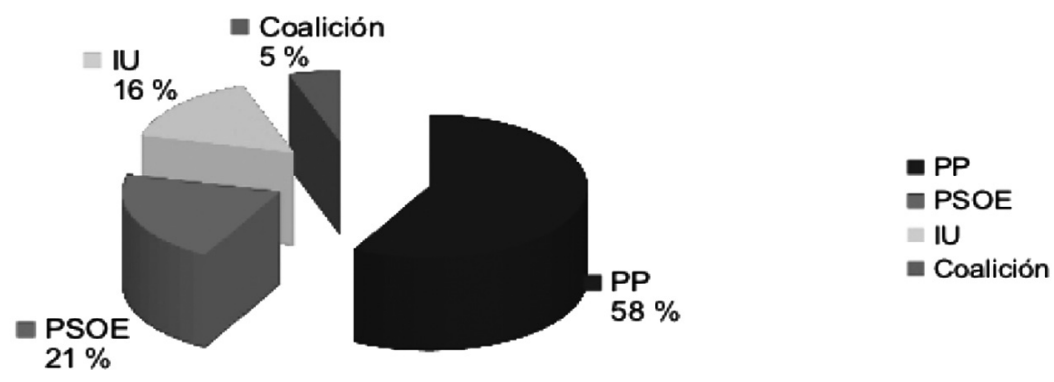

Fuente: elaboración propia

Figura 3. Partido en el gobierno en los municipios de Cádiz con licencia de radio local

Por otra parte, si observamos la figura 4, relativa a los municipios de la provincia de Cádiz en los que se han cerrado emisoras de radio, vemos que el $86 \%$ están gobernados por el PSOE, el 7 \% por el PP y el 7 \% por IU. Por lo tanto, tras el análisis comparativo de estos dos gráficos, no se sostiene la idea de que, en la actualidad, este tipo de medios esté siendo una prioridad política para partidos como el PSOE o IU. De los datos obtenidos en la provincia de Cádiz, vemos que sucede todo lo contrario: hay más estaciones de radio locales en los municipios gobernados por el PP y cierran más emisoras en municipios gobernados por el PSOE. 


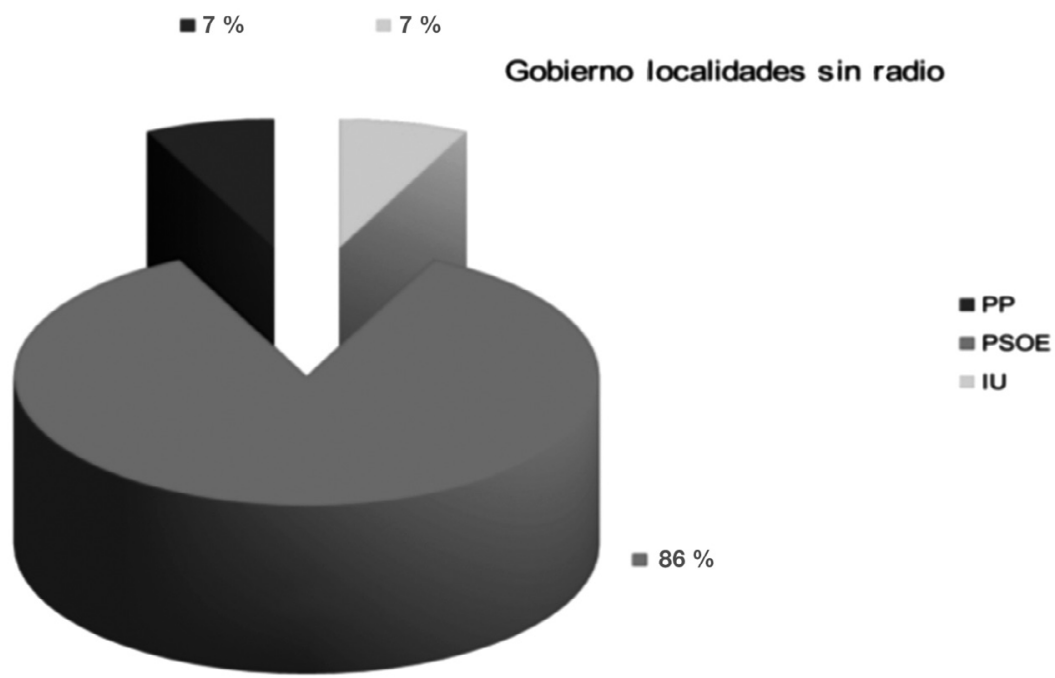

Fuente: elaboración propia

Figura 4. Partidos gobernantes en las localidades gaditanas con emisoras de radio cerradas

Estos datos también son susceptibles de ser releídos desde la perspectiva de una progresiva derechización del mapa político gaditano (una realidad constatable, también, a escala andaluza), en la medida en que el PP y otros partidos de la derecha han ido ganando espacio político en la provincia. De cualquier modo, aun partiendo de la base de que, en el origen, el impulso de las radios públicas locales contó con el apoyo de los partidos de izquierdas, estos medios no tuvieron el respaldo conseguido en otros países de nuestro entorno político. Así, si comparamos las medidas adoptadas por el gobierno de François Mitterand a su llegada al poder (1981) con las medidas impulsadas por el gobierno de Felipe González un año después (1982), comprobamos el diferente trato dado en los dos casos a los medios locales. En el caso francés, donde es más pertinente hablar de medios asociativos, el investigador Jean-Jacques Chéval reflexiona de este modo sobre la iniciativa del gobierno de Mitterand:

Estudiantes de radio, ambientalistas radiofónicos, Unión Radio, la radio pirata, radios «salvajes», como son llamadas en el momento. Las emisoras suelen ser militantes y aspiran a participar en una comunicación independiente, alternativa. La victoria de la unión de la izquierda y la elección de F. Mitterrand a la presidencia en mayo de 1981 dio lugar a la autorización de las radios libres, 
que se convirtieron en las radios locales privadas. Cientos, luego miles de estaciones locales, se crean en Francia. En 1982, la ley autoriza un plan claro y sencillo sobre a las estaciones de radio comunitarias (local de carácter no comercial) (Chéval, 2011: 120).

En el caso español, un año después de la llegada al poder de Mitterand, se producía la victoria del PSOE de Felipe González. Las esperanzas de quienes pensaron que las radios libres, comunitarias o ciudadanas iban a tener en España un tratamiento similar al recibido en Francia se difuminaron rápidamente. Como señalan investigadoras expertas en la materia,

En términos de las políticas de comunicación, por su parte, los años 80 marcaron el inicio de la transformación de la apertura europea sobre medios de comunicación públicos y privados, que también afectó a España. En este caso, la ley estatal desarrollada en medios como la radio y la televisión (como el Plan Técnico transitorio al servicio público de la radiodifusión sonora en frecuencia modulada en el año 1979 y la ley de televisión local sobre ondas terrestres 1995) también contribuyó a apoyar a las experiencias que habían surgido en la transición temprana, y más bien hizo hincapié en la privatización y la comercialización del espectro (Fleischman, Beachcomber y Sáez, 2009: 3).

En lo referente a la fase cualitativa de la investigación, analizamos ambos momentos (entrevistas en profundidad y análisis de contenido), apoyándonos en los 5 indicadores de Gumucio (2006).

Respecto al primer y segundo criterio (propiedad de la emisora; participación de la comunidad en la toma de decisiones) encontramos un impedimento legal que no permite que se cumplan. En cuanto a la propiedad, al ser un medio público, en nuestro caso local, pertenece al Ayuntamiento del municipio. Este hecho, que por definición cumple una función de apropiación -ya que lo público es de todos los ciudadanos- viene a desarrollar la función contraria, como muestra el segundo criterio.

El tipo de gestión de la emisora, determinado legalmente, mina la oportunidad de una verdadera apropiación del medio por parte de la ciudadanía porque ninguna de las tres tipologías - directa desde el ayuntamiento; a través del patronato de radio; a través de una empresa pública- permite una verdadera representación ciudadana en el proceso de toma de decisiones a la hora de diseñar las políticas de comunicación. Lo que ocurre respectivamente es que esta representación o no se da, o se da pero sin capacidad de voto, o viene condicionada políticamente. De modo que la representación y participación ciudadana, que debiera ser el contrapeso social en ese juego de fuerzas distinto 
a los intereses políticos y comerciales, no encuentra mecanismos para expresarse y ser escuchada.

Sobre el tercer criterio, la sostenibilidad de la emisora, a partir de los datos obtenidos vemos que se cumple a medias, es decir, existe un plan de sostenibilidad de la emisora a largo plazo. Esto también viene determinado legalmente, ya que la financiación de las emisoras públicas locales se realiza a través de los presupuestos de los Ayuntamientos. De modo que los medios locales dependen casi exclusivamente de cada Ayuntamiento y de los recursos económicos que este posea. Además de la financiación pública, las emisoras complementan esta con publicidad local. Destacar en este sentido a Radio Ubrique y Radio Trebujena, donde tienen presente el servicio que se presta a las empresas de la localidad y las promocionan, y la función de desarrollo local y económico que esto cumple.

A pesar de ello, la sostenibilidad de la emisora no atiende a una significación más orgánica de este concepto. No abogamos por una publicidad que condicione la independencia de la emisora, sino una que cumpla, como en estas dos emisoras, una función de desarrollo. Es decir, la sostenibilidad no hace tanto referencia a la viabilidad económica de la emisora como a su viabilidad a través de la integración entre ella y su entorno.

El cuarto y quinto criterio, que el $70 \%$ de los contenidos se generen localmente y la pertinencia lingüística y cultural, se cumplen con creces. Pero lo que hemos notado es que existen desviaciones respecto de lo que debería ser el enfoque participativo, producidas por los diferentes enfoques que predominan en las emisoras. Es decir, lo verdaderamente importante del contenido de la programación es que se mantenga un diálogo constante con los colectivos y asociaciones locales, así como con la ciudadanía, para definir la programación y los temas de interés, porque así se pone de manifiesto una verdadera preocupación de la emisora por constituirse en un elemento de desarrollo local. De nada sirve que el contenido esté centrado en la localidad cuando el enfoque de la emisora solo se preocupa por los ritmos culturales y festivos del municipio, porque de esta forma no se produce una apertura a las demandas ciudadanas sino que se silencian a través de un uso populista y evasivo de los medios de información.

Respecto a la pertinencia lingüística y cultural, nos apoyamos en el segundo momento del análisis cualitativo: el análisis de contenido, para apuntar que también encontramos divergencias en cuanto a la perspectiva participativa causadas por el enfoque temático de las emisoras: 
- La emisora de San Roque tiene un enfoque cultural y social, centrado en valores como la pluralidad y la diversidad. Se mantiene un diálogo constante con los colectivos y asociaciones locales para darles hueco en la programación. En Ubrique el enfoque es político-institucional y cultural. En esta emisora el diálogo con los colectivos y asociaciones no es tan constante ni tan determinante a la hora de diseñar la programación.

- La emisora Onda Jerez está enfocada a lo político-institucional y sobre todo a la cultura popular. En esta emisora no se produce un contacto fluido con las asociaciones locales, ni con los colectivos, y se pone de manifiesto un uso populista de lo cultural. En Trebujena se da un enfoque cultural y social. Esta emisora también mantiene un diálogo constante con los colectivos y asociaciones de la localidad, así como con el ciudadano de a pie, y se les da voz en la emisora.

El enfoque cultural y social es el más adecuado para los objetivos del servicio público, como es el caso de San Roque y Trebujena. Lo verdaderamente importante en este enfoque es que se mantenga un diálogo constante con los colectivos y asociaciones locales, así como con la ciudadanía, para definir la programación, porque esto revela una auténtica preocupación por constituirse en un dinamizador social.

La pertinencia lingüística y cultural de las emisoras se pone de manifiesto por la forma cercana y asequible de hacer partícipe a la audiencia y por los temas de interés local tratados, pero no cumple con los objetivos del enfoque participativo si no centra su atención y preocupación en que la ciudadanía tome conciencia de su situación. Si no centra su atención en los temas sociales, la pertinencia lingüística y cultural corre el riesgo de convertirse, centrando su atención en la cultura local, en un diálogo vacío que lleva a la autocontemplación, a la exaltación de la propia cultura y al estatismo, en vez de a la autorreflexión, a la crítica y al cambio.

\section{Conclusiones}

Tras el análisis realizado en este trabajo, y volviendo a la perspectiva de investigación planteada al inicio, consideramos que los medios de comunicación públicos y locales de Andalucía están siendo unos espacios privilegiados en los que identificar la calidad de la democracia impulsada por el poder local. En el actual contexto de crisis económico-financiera, social, política y, en 
última instancia, de paradigma de civilización, constatamos, en primer lugar, que los medios del tercer sector están siendo una víctima más de la crisis en marcha. El cierre de este tipo de emisoras y el recorte presupuestario al que se ven sometidas son, entre otros, indicadores de la nula prioridad que ocupa en las políticas municipales la articulación de espacios comunicativos liderados por la ciudadanía.

En segundo lugar, nuestro trabajo nos permite constatar que los medios del tercer sector son, en ocasiones, inéditos y viables en el sentido apuntado por Paulo Freire. Las radios públicas locales son posibilidades no exploradas (inéditas) y a la vez viables, en el actual contexto histórico, para llevar a cabo unas iniciativas comunicativas propias lideradas por parte del tercer sector de la comunicación. Para ello será necesario poner freno a las inercias y a las visiones reduccionistas procedentes tanto desde el poder local como desde el mercado, para que la lógica de la apropiación ciudadana sea la que predomine en estos medios.

En tercer lugar, el análisis cualitativo nos ha permitido identificar aquellas emisoras cuyas prácticas comunicativas se caracterizan por su atención al concepto de servicio público a la ciudadanía. En este sentido, destacamos las emisoras de San Roque y de Trebujena por su actitud de diálogo hacia los colectivos y asociaciones locales y por el espacio/voz que se les da. Como contrapunto encontramos emisoras como Onda Jerez, donde la lógica institucional se pone de manifiesto en el uso populista de la cultura local, desviando la atención hacia las fiestas y eventos populares como forma de exacerbar la identidad y los intereses y de establecer las preocupaciones de la ciudadanía.

Finalmente, añadir que el estudio sistemático de las buenas prácticas en la comunicación radial del tercer sector aportará elementos suficientes como para encontrar un espacio y unos rasgos propios desde los que la comunicación ciudadana se diferencia de los estilos y prácticas puestos en marcha por el Estado y el mercado. Asimismo, esta comunicación ciudadana será un indicador más a tener en cuenta en la necesaria revitalización de los procesos democráticos instituyentes.

\section{Referencias bibliográficas}

Cammaerts, B. (2009): «Community radio in the West: a legacy of struggle for survival in a state and capitalist controlled media environment», $G a$ zette, 71 (8): 635-654. 
Meda, M. (2010): «La ley UTECA y el tercer sector de la comunicación: comparativa internacional de las fallas de la legislación española audiovisual y respuesta de la sociedad civil», trabajo de fin de máster del Máster en Comunicación con Fines Sociales, estrategias y campañas de la Universidad de Valladolid (España), Universidad de Valladolid, Ediciones Discordia, recuperado de: https://uvadoc.uva.es/bitstream/10324/883/1/ TFM130-111222.pdf.

ChÉval, J. J. (2011): «El servicio público de la radio en Francia. Construcción e ilustraciones de un concepto original», en AA.vv. (ed.): Memorias de la $8^{a}$ Bienal Internacional de la Radio, México, Radio Educación, 119-125.

Chaparro, M. (2009): «Comunicación para el empoderamiento y comunicación ecosocial. La necesaria creación de nuevos imaginarios», Perspectivas de la Comunicación, 2 (1): 146-158.

Fleischman, L.; N. Reguero; C. Sáez (2009): «Políticas de comunicación y sustentabilidad del tercer sector de la comunicación: el caso catalán en el contexto español y europeo», Actas del Congreso Internacional de ULEPICC.

Gumucio-Dagron, A. (2006): «En Amman aman a AMARC», congreso mundial de radios comunitarias, recuperado de: http://www.bolpress.com/art. php?Cod=2006122601. 